\title{
Correction to: Combined therapeutic efficacy of carvacrol and X-radiation against 1,2-dimethyl hydrazine-induced experimental rat colon carcinogenesis
}

\author{
Sivaranjani Arivalagan ${ }^{1} \cdot$ Nisha Susan Thomas $^{1}$ - Balaji Chandrasekaran ${ }^{1} \cdot$ Vijay Mani $^{1} \cdot$ Aktarul Islam Siddique $^{1}$. \\ Thayalan Kuppsamy ${ }^{2} \cdot$ Nalini Namasivayam $^{1}$
}

Published online: 30 November 2021

(c) Springer Science+Business Media, LLC, part of Springer Nature 2021

\section{Correction to: \\ Molecular and Cellular Biochemistry (2015) 410:37-54 \\ https://doi.org/10.1007/s11010-015-2536-6}

In the original publication of the article, Fig. $2 b$ was published incorrectly. The correct version of Fig. 2 is provided in this correction.

The original article can be found online at https://doi.org/10.1007/ s11010-015-2536-6.

Nalini Namasivayam nalininam@yahoo.com

1 Department of Biochemistry and Biotechnology, Faculty of Science, Annamalai University, Annamalai Nagar, Tamil Nadu 608 002, India

2 Medical Physics Division, Dr. Kamakshi Memorial Hospital, No: 1, Radial Road, Pallikaranai, Chennai, Tamil Nadu 600 100, India 
a
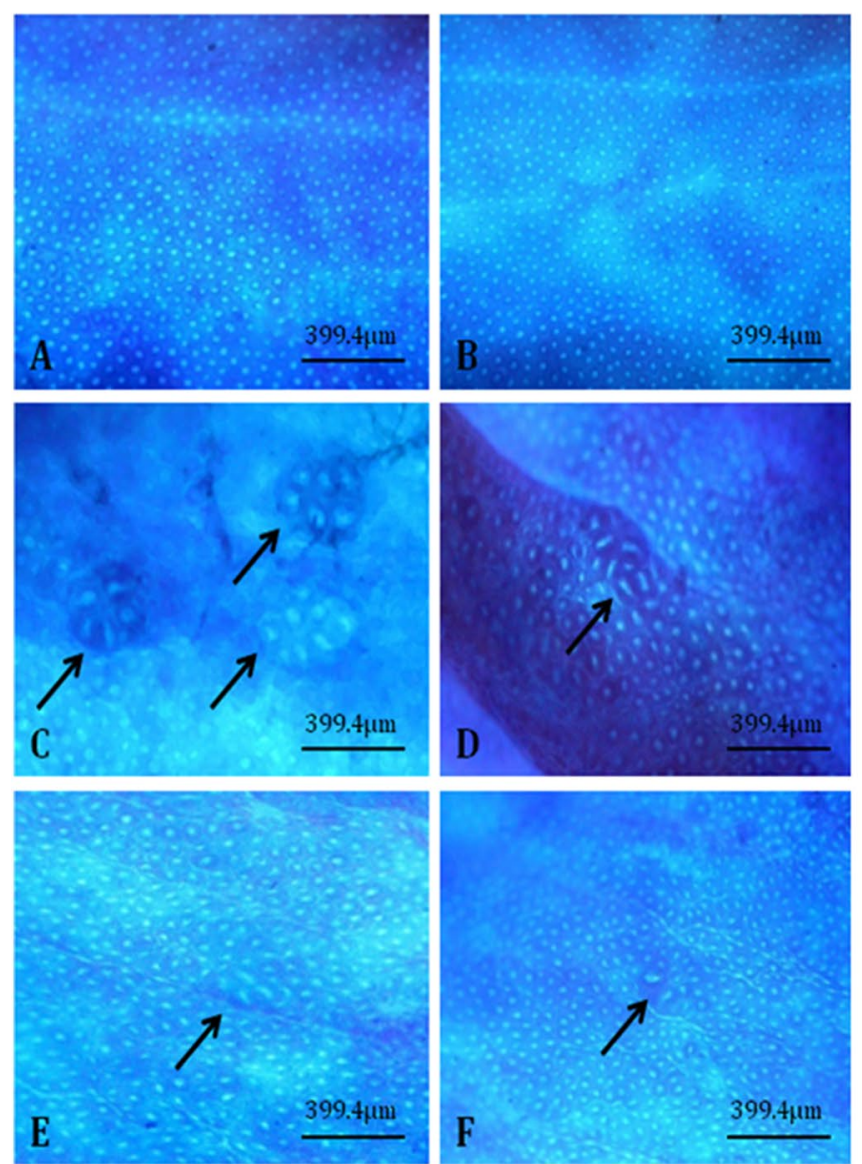

Fig. 2 a Topographical view of normal crypts and ACF in the colonic mucosa of experimental rats $(\times 10)$. A, B Colon of control and control $+\mathrm{CVC}$-treated rats show normal crypts. C Topographical view of ACF (arrows) with multiple (eighteen) crypts in the colon of a rat treated with DMH. D Topographical view of ACF (arrow) with eight crypts in the colon of a rat treated with $\mathrm{DMH}+\mathrm{X}$-radiation. E Topographical view of ACF (arrow) with four crypts in the colon of a rat treated with $\mathrm{DMH}+\mathrm{CVC}$. F Topographical view of ACF (arrow) with two crypts in the colon of a rat treated with $\mathrm{DMH}+\mathrm{CVC}+\mathrm{X}$-radiation. $\mathbf{b}$ Topographical view of dysplastic ACF in the colonic mucosa of experimental animals $(\times 20)$. A: Normal

Publisher's Note Springer Nature remains neutral with regard to jurisdictional claims in published maps and institutional affiliations.

\section{b}


crypts in the unsectioned colon of control rats. B Normal crypts in the unsectioned colon of the rats supplemented with CVC alone. $\mathbf{C}$ DACF (arrow) with thirteen crypts resist methanol, decolouration after methylene blue staining in the unsectioned colon of the rat treated with DMH alone. D DACF (arrow) with seven crypts in the unsectioned colon of DMH-treated rat exposed to radiation alone. $\mathbf{E}$ DACF (arrow) with four crypts in the unsectioned colon of DMHtreated rat supplemented with CVC. F DACF (arrow) with three crypts in the unsectioned colon of DMH-treated rat treated with both CVC and X-radiation 